\title{
Energetics of hydrogen impurities in aluminum and their effect on mechanical properties
}

\author{
Gang Lu, Daniel Orlikowski, Ickjin Park, Olivier Politano, and Efthimios Kaxiras \\ Department of Physics and Division of Engineering and Applied Sciences, Harvard University, Cambridge, Massachusetts 02138
}

(Received 6 September 2001; published 3 January 2002)

\begin{abstract}
The effects of hydrogen impurities in the bulk and on the surface of aluminum are theoretically investigated. Within the framework of density-functional theory, we have obtained the dependence on $\mathrm{H}$ concentration of the stacking fault energy, the cleavage energy, the $\mathrm{Al} / \mathrm{H}$ surface energy, and the $\mathrm{Al} / \mathrm{H} / \mathrm{Al}$ interface formation energy. The results indicate a strong dependence of the slip energy barrier in the [ $\overline{2} 11]$ direction, the cleavage energy in the [111] direction and the $\mathrm{Al} / \mathrm{H} / \mathrm{Al}$ interface formation energy, on $\mathrm{H}$ concentration and on tension. The dependence of the $\mathrm{Al} / \mathrm{H}$ surface energy on $\mathrm{H}$ coverage is less pronounced, while the optimal $\mathrm{H}$ coverage is $\leqslant 0.25 \mathrm{ML}$. The calculated activation energy for diffusion between high-symmetry sites in the bulk and on the surface is practically the same, $0.167 \mathrm{eV}$. From these results, we draw conclusions about the possible effect of $\mathrm{H}$ impurities on mechanical properties and, in particular, on their role in embrittlement of $\mathrm{Al}$.
\end{abstract}

DOI: 10.1103/PhysRevB.65.064102

PACS number(s): 61.72.Qq, 61.72.Nn, 66.30.Fq

\section{INTRODUCTION}

In many technological applications of advanced materials a crucial aspect of performance is the control of environmental effects, such as the presence of impurities. One such impurity is hydrogen, which pervades most metals and degrades their performance. ${ }^{1}$ The interactions of $\mathrm{H}$ with lattice imperfections, such as dislocations, stacking faults, surfaces, and microcracks, dominate its influence on the mechanical properties of a material. However, these interactions are far less well understood at a fundamental level than the behavior of $\mathrm{H}$ in perfect crystals. Therefore, atomistic studies based on parameter-free, $a b$ initio calculations are of great interest because they can provide accurate energetics for the various $\mathrm{H}$-defect complexes and probe the microscopic physics responsible for the macroscopic behavior. The impurity-defect energetics are not only interesting by themselves, they can also be incorporated in more sophisticated models in order to make quantitative predictions for the macroscopic properties of solids, in what has become known as multiscale simulations of materials. ${ }^{2,3}$

The present study is motivated by the desire to shed light into $\mathrm{H}$ embrittlement of $\mathrm{Al}$ from an electronic structure point of view. Experimentally, the presence of $\mathrm{H}$ in $\mathrm{Al}$ is associated with enhanced dislocation activity that, perhaps paradoxically, leads to a brittle rupture failure. ${ }^{1,4,5}$ Theoretically, it has been shown recently within the framework of the PeierlsNabarro model, that the presence of $\mathrm{H}$ in $\mathrm{Al}$ can dramatically enhance dislocation mobility and inhibit dislocation cross slip. ${ }^{2}$ However, the underlying atomic bonding features that give rise to such dislocation behavior have not been explored. In this paper, we show how $\mathrm{H}$ can change the nature of chemical bonding in Al leading to so-called hydrogenenhanced local plasticity (HELP). ${ }^{1,2}$ Moreover, we show that the cleavage energy, which represents the ultimate resistance to fracture, can be considerably reduced by $\mathrm{H}$.

Another important aspect of $\mathrm{H}$ behavior in $\mathrm{Al}$ is the thermodynamics of $\mathrm{H}$ in bulk $\mathrm{Al}$ and on its (111) surface, and the corresponding diffusion constants. The stability and mobility of $\mathrm{H}$ impurities in $\mathrm{Al}$ play an important role in HELP and $\mathrm{H}$ embrittlement of $\mathrm{Al}$. For example, it is observed experimen- tally that HELP occurs only when the thermal diffusion of $\mathrm{H}$ in the lattice is fast enough to follow the motion of dislocations. ${ }^{1}$ Accordingly, we calculate the diffusion energy barriers and the diffusion constants for $\mathrm{H}$ in the bulk and on the (111) surface of Al. We also examine the $\mathrm{H}$ diffusion process under uniform tensile strain to simulate the behavior at the dislocation core or near the crack tip region.

The outline of this paper is as follows: we briefly describe our computational methodology in Sec. II. In Sec. III, we present the detailed results for the energetics of various defect structures involving $\mathrm{H}$ in the bulk and on the (111) surface of $\mathrm{Al}$ and attempt to understand some of the energetics from an electronic structure point of view. We discuss the physical consequences of our results on the mechanical properties of $\mathrm{Al}$ and conclude in Sec. IV.

\section{METHODOLOGY}

The $a b$ initio calculations we performed are based on density-functional theory with the VASP (Vienna Ab-initio Simulation Package) implementation ${ }^{6}$ and ultrasoft pseudopotentials. ${ }^{7}$ The Ceperley-Alder exchange-correlation potentials ${ }^{8}$ paramtrized by Perdew-Zunger ${ }^{9}$ were used in the present calculations. We have performed $k$-point convergence studies in all cases using a uniform Monkhorst-Pack scheme. ${ }^{10}$ From these studies, we have determined that a grid consisting of $16 \times 16 \times 16$ divisions in the Brillouin zone of the primitive unit cell of bulk fcc Al, appropriately scaled for larger unit cells, is adequate for good convergence. The kinetic-energy cutoff of $130 \mathrm{eV}$ for pure $\mathrm{Al}$ yields wellconverged results, whereas a higher cutoff of $350 \mathrm{eV}$ is needed in the presence of $\mathrm{H}$ atoms. We have also introduced a smearing of the Fermi surface by a temperature of $25 \mathrm{meV}$. With these computational parameters, the calculated lattice constant for bulk fcc $\mathrm{Al}$ is $a=3.99 \AA$ and the bulk modulus is $B=83.2 \mathrm{GPa}$, determined by a Birch-Murnaghan fit to the energy vs volume curve. ${ }^{11}$ These values compare well with experimental values ${ }^{12}$ of $a=4.05 \AA$ and $B=76.9 \mathrm{GPa}$, respectively.

We next turn our attention to the atomic structures used to represent the physical systems of interest. In Fig. 1, we show 


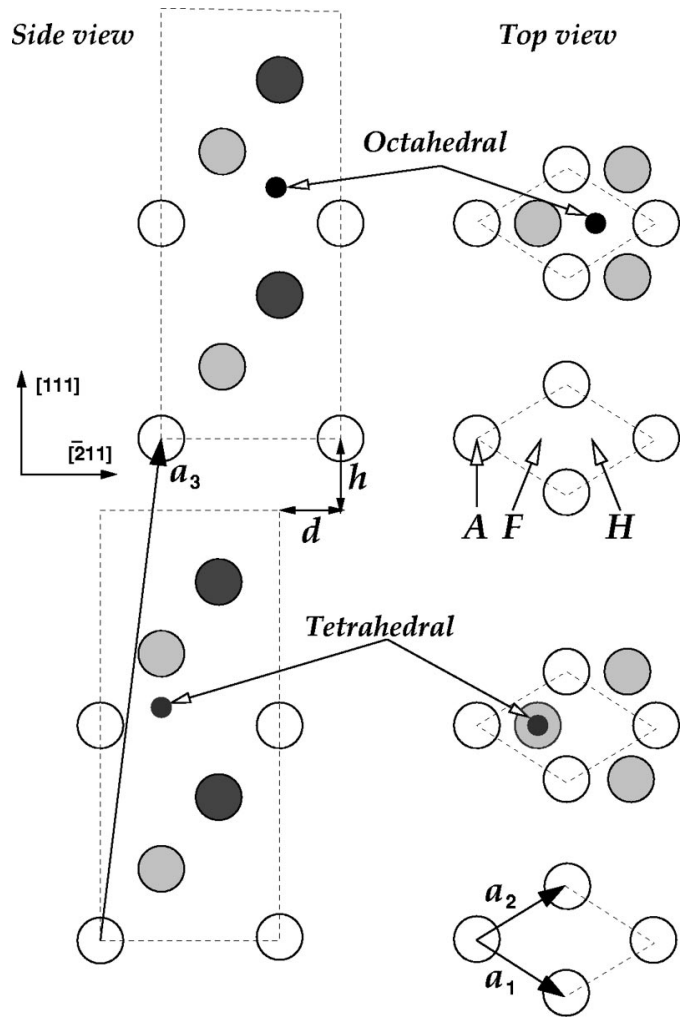

FIG. 1. Schematic representation of the geometry used in the total-energy calculations. The basic supercell is shown in side and top views. The dashed lines outline the undistorted supercell. It consists of six layers in the [111] direction and has repeat vectors in the (111) plane equal to the ideal crystal primitive lattice vectors, denoted by $\mathbf{a}_{1}, \mathbf{a}_{2}$. The third vector $\mathbf{a}_{3}$ is along the [111] direction in the undistorted cell. Distortions of the cell by adding to $\mathbf{a}_{3}$ components in the $[\overline{2} 11]$ direction (denoted by $d$, given in units of $a / \sqrt{6}$ ), or in the [111] direction (denoted by $h$, given in units of $a / \sqrt{3}$ ), lead to configurations relevant to the generalized stacking fault energy surface or to the cleavage energy; such a distortion for $d=1, h=1$ is illustrated. The large white, gray, and black circles indicate the positions of the $\mathrm{Al}$ atoms; all $\mathrm{Al}$ atoms contained in a unit cell are shown in the side view, but only selected planes of atoms are shown in the top view. The smaller black circle indicates the highsymmetry positions of the $\mathrm{H}$ atom in the bulk (tetrahedral and octahedral), and on the (111) surface ( $F$ for the fcc site, $H$ for the hcp site and $A$ for the atop site).

the basic supercell that consists of six Al layers in the [111] direction. In all the calculations, we used the same number of layers in this direction to represent the (111) surface or the interface between two semi-infinite slabs. We have used this supercell and multiples or distortions of it to calculate the generalized stacking fault energy curve and the surface and interface energies. The unit cell with periodicity in the (111) plane equal to that of the bulk crystal will be referred to as the $1 \times 1$ cell. Multiples of the in-plane vectors, denoted as $\mathbf{a}_{1}$ and $\mathbf{a}_{2}$ in Fig. 1, were used to create larger supercells for studying the effects of $\mathrm{H}$ concentration. We have used ( $q \mathbf{a}_{1}$ $\times q \mathbf{a}_{2}$ ) multiples of the basic cell with $q=1, \sqrt{3}$, and 2 . In each supercell we included one $\mathrm{H}$ atom. These configurations correspond to $\mathrm{H}$ concentrations in the bulk of 14.3, 5.3, and 4.0 at. \%, respectively. On the surface, they correspond to $\mathrm{H}$ monolayer (ML) coverages of $\Theta=1.0,0.333$, and $0.25 \mathrm{ML}$, respectively. We also report a single surface calculation with a $4 \times 4$ unit cell, corresponding to $\mathrm{H}$ coverage $\Theta$ $=0.0625 \mathrm{ML}$, in order to establish the value of the $\mathrm{H} / \mathrm{Al}$ surface energy in the low coverage limit. The $\mathrm{H}$ atom in each supercell was placed at the high-symmetry interstitial sites, identified as the tetrahedral, fourfold coordinated $(T)$ or octahedral, sixfold coordinated $(O)$ position in the undistorted bulk configuration, or as the fcc $(F)$, hcp $(H)$, and atop $(A)$ sites on the (111) surface, all shown in Fig. 1. Calculations with the $\mathrm{H}$ atom in positions between the high-symmetry sites in the bulk and on the surface were used to obtain diffusion energy barriers.

Distorting the $q \times q$ supercell by increasing the horizontal or vertical components of $\mathbf{a}_{3}$, the lattice vector that in the undistorted case lies along the [111] direction, produces configurations that generate the generalized stacking fault energy or the cleavage energy. These two distortions are referred to as $d$ and $h$, respectively, and are given in their natural units of $a / \sqrt{6}$ and $a / \sqrt{3}$. In these units, $d=1$ corresponds to the intrinsic stacking fault configuration, $d=2$ corresponds to the so-called run-on configuration (in which two $\mathrm{Al}$ atoms on either side of the slip plane are exactly above and below each other), and $d=3$ corresponds to another ideal configuration identical to $d=0$. Similarly, $h=1$ corresponds to a separation between the two slabs equivalent to a missing (111) layer.

For each of these configurations, all atoms except the innermost two layers of the Al slab were fully relaxed via the conjugate-gradient method, so that the magnitude of the calculated forces on the atoms was less than $0.03 \mathrm{eV} / \AA$. For the calculations of the energy barriers for diffusion, the coordinates of the $\mathrm{H}$ atom are held fixed, either in all three directions for bulk diffusion, or in the lateral surface directions for surface diffusion. For the calculation of the generalized stacking fault energies using the distorted bulk supercells (see below), the $\mathrm{H}$ atom was placed initially close to the interpolated tetrahedral or octahedral positions and allowed to relax to the nearest local energy minimum. We report energy differences between various configurations in $\mathrm{eV}$ and surface energies in $\mathrm{J} \mathrm{m}^{-2}$, in order to comply with conventions in the literature and make our results easily comparable to other published work.

\section{ENERGETICS OF H IMPURITIES IN AI}

\section{A. Generalized stacking fault energies}

The generalized stacking fault (GSF) energy, denoted by $\gamma_{\mathrm{GSF}}$, is defined as the energy cost per unit area for sliding two semi-infinite slabs relative to each other along a particular plane by a certain vector $\mathbf{d}$. The energy surface generated by spanning the allowed values of $\mathbf{d}$ contains several important features relevant to the mechanical properties of solids and, in particular, to their brittle vs ductile behavior. For an fcc metal such as Al the most interesting portion of the GSF energy surface is the path along the $[\overline{2} 11]$ direction on the (111) plane. This path includes both the intrinsic stacking fault as well as the unstable stacking fault, corresponding to 


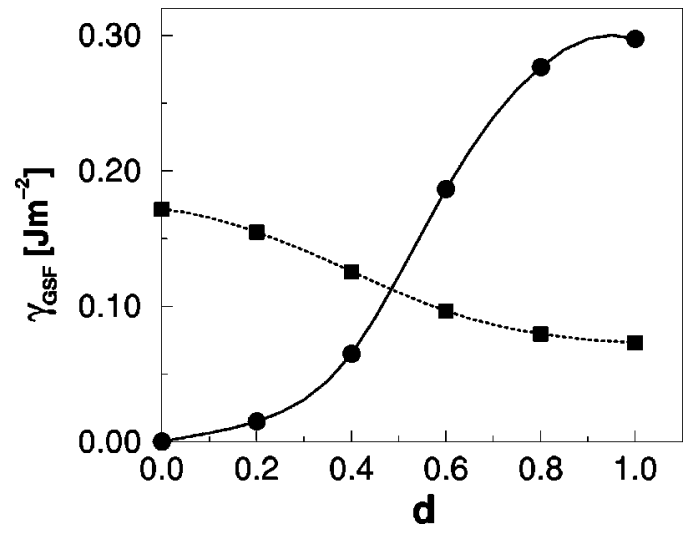

FIG. 2. Generalized stacking fault energy $\gamma_{\mathrm{GSF}}$ for $\mathrm{Al}$ with $\mathrm{H}$ impurities at zero tension opening, $h=0$, as a function of the slip $d$ in the $[\overline{2} 11]$ direction for the $1 \times 1$ supercell, corresponding to $\mathrm{H}$ concentration of 14.3 at. \%: circles represent the energy for the tetrahedral site, squares for the octahedral site (lines are fits intended as guide to the eye).

$d=1$ and 0.6 , respectively. The intrinsic stacking fault energy, denoted as $\gamma_{\mathrm{sf}}$, along with the elastic properties of the material, determine the separation distance between partial dislocations ${ }^{12}$ that controls the mobility of the dislocations. ${ }^{13}$ The unstable stacking fault energy, denoted as $\gamma_{\text {us }}$, represents the energy barrier for dislocation nucleation from a crack tip, which is related to the tendency for brittle or ductile behavior of the material. ${ }^{14}$

The values of $\gamma_{\mathrm{GSF}}$ for pure $\mathrm{Al}$ have been published elsewhere. ${ }^{15,16}$ In the present work we have repeated these calculations to obtain a consistent set of numbers with the computational parameters and methodology adopted here. The values of the important configurations obtained by the present calculations are: $\gamma_{\mathrm{us}}=0.182 \mathrm{~J} \mathrm{~m}^{-2}$, and $\gamma_{\mathrm{sf}}$ $=0.134 \mathrm{~J} \mathrm{~m}^{-2}$, both for $h=0$. Including tension opening $(h \neq 0)$, reduces these values dramatically. For example, for $h=0.1$, we obtained $\gamma_{\text {us }}=0.094 \mathrm{~J} \mathrm{~m}^{-2}$, a $50 \%$ reduction and $\gamma_{\mathrm{sf}}=0.092 \mathrm{~J} \mathrm{~m}^{-2}$, a $30 \%$ reduction.

The values of $\gamma_{\mathrm{GSF}}$ in the presence of $\mathrm{H}$ depend on the position of the $\mathrm{H}$ atom in the lattice and the $\mathrm{H}$ concentration. We next examine these two contributions separately. We consider first our findings for the highest $\mathrm{H}$ concentration, 14.3 at. \%, which corresponds to one $\mathrm{H}$ atom in a $1 \times 1$ supercell. The results, shown in Fig. 2, indicate that there is a crossover at approximately $d=0.5$ in site preference for the $\mathrm{H}$ atom. This crossover in site preference significantly reduces the unstable stacking energies to $\gamma_{\mathrm{us}}=0.097 \mathrm{~J} \mathrm{~m}^{-2}$, a $\sim 50 \%$ re- duction, and the intrinsic stacking fault energy to $\gamma_{\mathrm{sf}}$ $=0.073 \mathrm{~J} \mathrm{~m}^{-2}$, also a $\sim 50 \%$ reduction. The reason for such energy reduction is that the volume available for the interstitial $\mathrm{H}$ atom situated at the original octahedral site decreases during the slip, while it increases at the tetrahedral site. The effects of tension on $\gamma_{\mathrm{GSF}}$ in the presence of $\mathrm{H}$ were also calculated and found to be very similar to those for pure $\mathrm{Al}$, as far as the relative energy decrease is concerned.

To study the dependence of $\gamma_{\text {us }}$ and $\gamma_{\mathrm{sf}}$ on $\mathrm{H}$ concentration, we have computed these energies for several $\mathrm{H}$ concentrations. The results are given in Table I. The general trend for both energies is to increase with decreasing $\mathrm{H}$ concentration. An exception to the trend is the highest $\mathrm{H}$ concentration at 14.3 at. $\%$ (from the $1 \times 1$ supercell), which has higher values for $\gamma_{\mathrm{us}}$ and $\gamma_{\mathrm{sf}}$ than the next lower $\mathrm{H}$ concentration of 5.3 at. \% (from the $\sqrt{3} \times \sqrt{3}$ supercell). We believe that this has to do with the fact that in the $1 \times 1$ supercell at fixed volume, the optimal ionic bonding distances between $\mathrm{H}$ and $\mathrm{Al}$ atoms cannot be satisfied and, therefore, the system cannot attain a structure with a reasonably low energy. To investigate this conjecture, we have also examined thoroughly the effect of volume relaxation in the $1 \times 1$ supercell. We find that in this supercell there is actually a low-energy configuration at the run-on position, $d=2$, which is lower in energy by $0.615 \mathrm{~J} \mathrm{~m}^{-2}$ than the undistorted configuration of $d=0$, and involves an increase in the volume by $18 \%$. In this configuration, the $\mathrm{H}$ atom lies exactly between the two $\mathrm{Al}$ atoms on either side of the slip plane, forming strong ionic bonds across the interface, which compensates the energy loss due to the distortion of the $\mathrm{Al}$ lattice.

\section{B. Cleavage energy}

Another important energy for the mechanical behavior of a solid is the cleavage energy $\gamma_{\mathrm{cl}}$, defined as the energy cost per unit area to separate the solid into two semi-infinite halves by creating two surfaces. For the pure $\mathrm{Al}$ case, $\gamma_{\mathrm{cl}}$ $=2 \gamma_{s}$, with $\gamma_{s}$ the energy of the newly created surface. We have calculated the cleavage energy with the various supercells as the vertical component of $\mathbf{a}_{3}$ is increased up to $h$ $=4$, corresponding to a separation of about $9 \AA$. The $\mathrm{H}$ atom was placed at the energetically preferred tetrahedral site. With the introduction of $\mathrm{H}$ to the system, the cleavage energy dramatically decreases by as much as $50 \%$ at $\mathrm{H}$ concentration of 14.3 at. \% (see Table I). The decrease of the cleavage energy is approximately proportional to the $\mathrm{H}$ concentration.

In order to elucidate the origin of the reduction in the GSF energy and the cleavage energy in the presence of $\mathrm{H}$, we

TABLE I. Unstable stacking energy $\gamma_{\mathrm{us}}$, stacking fault energy $\gamma_{\mathrm{sf}}$, and cleavage energy $\gamma_{\mathrm{cl}}$ for the H/Al system as a function of $\mathrm{H}$ concentration without volume relaxation. The ratio $\gamma_{\mathrm{cl}} / \gamma_{\mathrm{us}}$ is also included.

\begin{tabular}{cccccc}
\hline \hline Supercell & at. \% H & $\begin{array}{c}\gamma_{\mathrm{us}} \\
\left(\mathrm{J} \mathrm{m}^{-2}\right)\end{array}$ & $\begin{array}{c}\gamma_{\mathrm{sf}} \\
\left(\mathrm{J} \mathrm{m}^{-2}\right)\end{array}$ & $\begin{array}{c}\gamma_{\mathrm{cl}} \\
\left(\mathrm{J} \mathrm{m}^{-2}\right)\end{array}$ & $\gamma_{\mathrm{cl}} / \gamma_{\mathrm{us}}$ \\
\hline $1 \times 1$ & 14.3 & 0.097 & 0.073 & 0.930 & 9.6 \\
$\sqrt{3} \times \sqrt{3}$ & 5.3 & 0.089 & 0.071 & 1.611 & 18.1 \\
$2 \times 2$ & 4.0 & 0.136 & 0.074 & 1.680 & 15.6 \\
$1 \times 1$ & 0.0 & 0.182 & 0.134 & 1.934 & 10.6 \\
\hline \hline
\end{tabular}




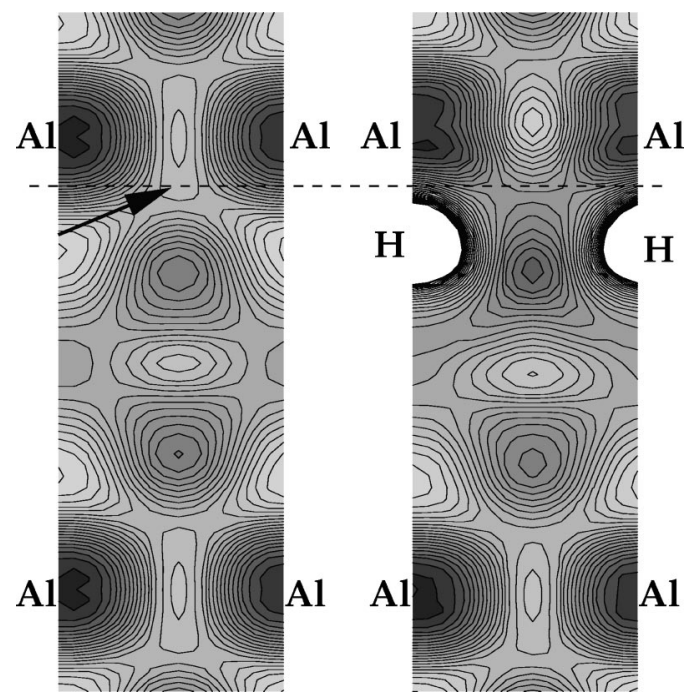

FIG. 3. Bonding valence charge density on the $(\overline{1} 2 \overline{1})$ plane for pure $\mathrm{Al}$ (left) and $\mathrm{Al}+\mathrm{H}$ (right) systems at $d=0$. The horizontal direction is $[10 \overline{1}]$ and the vertical direction is [111]. The fictitious slip plane is shown as a dashed line and the positions of atoms are indicated by the corresponding labels. The arrow indicates the buildup of valence charge density corresponding to covalent bonding across the slip plane.

examine the bonding charge density on the $(\overline{1} 2 \overline{1})$ plane for pure $\mathrm{Al}$ and for $\mathrm{Al}+\mathrm{H}(14.3$ at. \%) at $d=0$, which is shown in Fig. 3. The bonding charge density is defined as the difference between the valence charge density in the solid and the superposition of neutral atomic valence charge densities placed at the lattice sites. The positive (negative) bonding charge density represents the net gain (loss) of charge as the atoms are brought together to form the solid. The contour graph is shaded in such a way that regions with higher value of charge density are lighter. By examining the bonding charge density of $\mathrm{Al}$ and $\mathrm{Al}+\mathrm{H}$, we find extended covalent bonding (indicated by the arrow) in $\mathrm{Al}$ across the slip plane, which is dramatically weakened in the presence of $\mathrm{H}$. In fact, the $\mathrm{H}$ atom depletes the $\mathrm{Al}$ bonding charge from the interstitial region and the regions across the slip plane to form ionic bonding between the $\mathrm{H}$ sites and the nearest $\mathrm{Al}$ sites above it. As a consequence, the cohesive strength across the slip plane is reduced by the presence of $\mathrm{H}$, giving rise to the lower cleavage energies. More importantly, since the strength of the ionic bonding between the positively charged Al plane and negatively charged $\mathrm{H}$ plane is not sensitive to the relative sliding between the two planes, the sliding energy barrier is greatly reduced, and the GSF energy surface becomes much smoother $^{2}$ in the presence of $\mathrm{H}$. This is contrasted to the pure $\mathrm{Al}$ case, where the covalent bonding among $\mathrm{Al}$ atoms across the slip plane is very sensitive to the local bonding distortions and consequently the GSF energy is higher and has more pronounced features. ${ }^{15}$ Although these calculations concern $\mathrm{Al}$, we believe that the results are also applicable to other metals whose electronegativity is lower than $\mathrm{H}$.

In Table I, we also give the ratio of $\gamma_{\mathrm{cl}}$ to $\gamma_{\mathrm{us}}$ for the various $\mathrm{H}$ concentrations with pure $\mathrm{Al}$ as the reference point. The value of this ratio is indicative of the tendency of the material to exhibit brittle or ductile behavior. ${ }^{14}$ From this comparison, we infer that at modest $\mathrm{H}$ concentrations the system has increased ductility, which is consistent with the experimental observations concerning HELP, ${ }^{1}$ but at the highest $\mathrm{H}$ concentration considered the system may become less ductile. The anomaly of the $\gamma_{\mathrm{cl}} / \gamma_{\mathrm{us}}$ ratio at the highest $\mathrm{H}$ concentration is related to the anomalous behavior of $\gamma_{\mathrm{us}}$ at this concentration, as noted earlier.

\section{Absorption and diffusion of $\mathbf{H}$ in bulk Al}

In view of the importance of the thermodynamics of $\mathrm{H}$ in bulk $\mathrm{Al}$ and the kinetics of $\mathrm{H}$ transport in the presence of defects, we have performed additional calculations for the energetics of $\mathrm{H}$ absorption and the $\mathrm{H}$ diffusion energy barrier in bulk Al. For these calculations, we employed a 32-atom supercell of the bulk crystal, which is a multiple of the conventional simple cubic cell by a factor of 2 in each direction (hence, we refer to it as the $2 \times 2 \times 2$ supercell). We have investigated the two high-symmetry interstitial sites of a single $\mathrm{H}$ impurity, which corresponds to a $\mathrm{H}$ concentration of 3.03 at. \%. The absorption energy $E_{\text {ab }}$ was obtained with reference to the cohesive energy of crystalline fcc $\mathrm{Al} E_{c}(\mathrm{Al})$, and using a gas of $\mathrm{H}_{2}$ molecules, whose binding energy is $E_{b}\left(\mathrm{H}_{2}\right)$, as a reservoir for the $\mathrm{H}$ atoms:

$$
E_{\mathrm{ab}}=E_{c}(\mathrm{H} / \mathrm{Al})-N_{\mathrm{sc}} E_{c}(\mathrm{Al})-\frac{1}{2} E_{b}\left(\mathrm{H}_{2}\right),
$$

where $E_{c}(\mathrm{H} / \mathrm{Al})$ is the calculated cohesive energy of the $\mathrm{Al}$ supercell configuration with one $\mathrm{H}$ impurity and $N_{\mathrm{sc}}$ is the number of $\mathrm{Al}$ atoms in the supercell $\left(N_{\mathrm{sc}}=32\right.$ in the present case). The binding energy of the $\mathrm{H}_{2}$ molecule was calculated to be $-6.697 \mathrm{eV}$ in vacuum, using a cubic cell with side equal to $24 \AA$, and the same computational parameters as for the $\mathrm{H} / \mathrm{Al}$ system. We find $E_{\mathrm{ab}}^{(T)}=-0.222 \mathrm{eV}$ for the tetrahedral site and $E_{\mathrm{ab}}^{(O)}=-0.152 \mathrm{eV}$ for the octahedral site. These results show that the incorporation of $\mathrm{H}$ in bulk $\mathrm{Al}$, starting with an $\mathrm{Al}$ crystal and $\mathrm{H}_{2}$ gas, is a thermodynamically exothermic process. Therefore, the $\mathrm{H}$ impurity is thermodynamically stable in bulk Al.

Having established the stability of $\mathrm{H}$ in bulk $\mathrm{Al}$, we investigated the mobility of $\mathrm{H}$ in the Al lattice. As alluded to earlier, the diffusion rate of $\mathrm{H}$ in the lattice determines whether H-enhanced local plasticity can occur or not. HELP takes place only when the diffusion of $\mathrm{H}$ atoms is fast enough to allow them to redistribute around the core of a moving dislocation (dynamic trapping) and thereby continuously minimize the system energy. ${ }^{1} \mathrm{H}$ diffusion can also affect the kinetics of crack propagation, the strain rate dependence of $\mathrm{H}$ embrittlement, and the rate of hydride formation. ${ }^{17}$ Since $\mathrm{H}$ jumps between nearby interstitial sites in bulk $\mathrm{Al}$, we calculated the activation energy of $\mathrm{H}$ diffusion between the closest tetrahedral and octahedral sites. In Fig. 4, we show the energy as a function of relative position of the $\mathrm{H}$ atom moving between the tetrahedral (0 and 2$)$ and the octahedral site (1). Another special site is the midpoint between the tetrahedral and the octahedral sites. We define the energy of the midpoint and of the octahedral site relative to the tetrahedral site as $\varepsilon_{1}$ and $\varepsilon_{2}$, respectively. The diffusion energy barrier for unstrained bulk $\mathrm{Al}$ is $\varepsilon_{b}=0.167 \mathrm{eV}$, which 


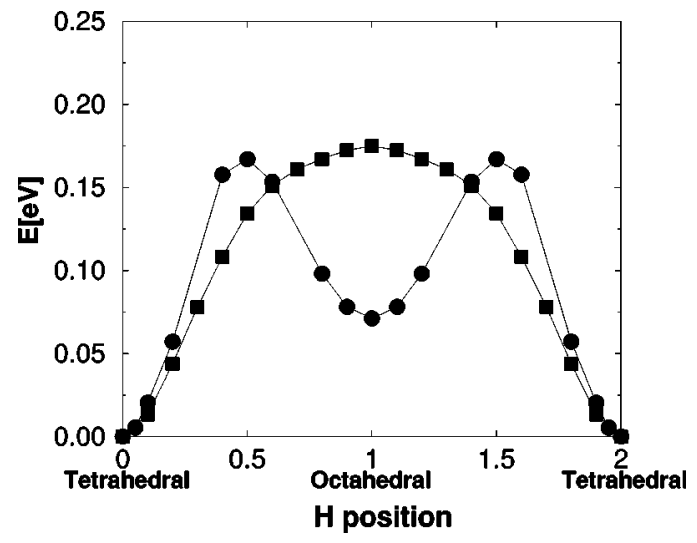

FIG. 4. Relative energy for motion of a $\mathrm{H}$ atom from the tetrahedral position (0) to the octahedral position (1) in the 32-atom bulk supercell. Circles represent the energy without tensile strain, and squares correspond the energy with $5 \%$ tensile strain.

compares well with the experimental value ${ }^{18}$ of $0.168 \mathrm{eV}$. The energy barrier configuration happens to coincide with the midpoint between the octahedral and tetrahedral sites in the unstrained crystal, that is $\varepsilon_{b}=\varepsilon_{1}$. Since we are also interested in the interaction of $\mathrm{H}$ with lattice imperfections, such as dislocations, microcracks, etc., we considered how $\mathrm{H}$ diffusion is affected by the presence of such defects. A simple way to simulate this effect is to apply strain to the system. In this study, we concentrate on how tensile strain affects $\mathrm{H}$ diffusion in $\mathrm{Al}$, since this is the type of strain field usually found around an edge dislocation or a crack tip under model I loading, which are both relevant to $\mathrm{H}$ embrittlement of Al. For the range of tensile strains studied, the tetrahedral site has the lowest energy. When the hydrostatic tensile strain is small $(\leqslant 3 \%)$, the energy barrier is located at the midpoint between the tetrahedral and the octahedral sites, i.e., $\varepsilon_{b}$ $=\varepsilon_{1}$. Moreover, we find that for small strain the diffusion energy barrier drops monotonically as the strain increases, and is reduced to $0.150 \mathrm{eV}$ for $3 \%$ expansion. This result is important because it shows that not only $\mathrm{H}$ prefers to stay in slightly enlarged interstitial regions such as dislocation cores and crack tips, but that it can also move more easily within such regions. On the other hand, if the tensile strain is large $(>3 \%)$, the octahedral site becomes energetically unstable and represents the energy maximum, where the energy barrier for diffusion is located, that is $\varepsilon_{b}=\varepsilon_{2}$. One example for such diffusion energy profile is shown in Fig. 4, corresponding to 5\% strain. The instability of the octahedral site arises from the unfavorable bond length between $\mathrm{H}$ and $\mathrm{Al}$ atoms, $2.1 \AA$, which is much larger than the preferred ionic bond length of about $1.8 \AA$. The values of $\varepsilon_{1}$ and $\varepsilon_{2}$ as a function of tensile strain are summarized in Table II. Noticing that the tetrahedral site is always energetically favorable regardless of the strain, we also calculated the relative energy of $\mathrm{H}$ at the midpoint of the direct line between two adjacent tetrahedral sites defined as $\varepsilon_{3}$ as a function of tensile strain. We find this energy difference to be always higher than the corresponding values of $\varepsilon_{1}$ and $\varepsilon_{2}$. Therefore, we have confirmed that the tetrahedral-octahedral-tetrahedral sequence is the
TABLE II. Energy of special points for $\mathrm{H}$ diffusion in bulk $\mathrm{Al}$, relative to the tetrahedral position: $\varepsilon_{1}$ is the energy of the midpoint between the tetrahedral and octahedral positions. $\varepsilon_{2}$ is the energy of the octahedral position and $\varepsilon_{3}$ is the energy of the midpoint between adjacent tetrahedral sites. The asterisks denote the energy barrier for diffusion.

\begin{tabular}{cccc}
\hline \hline $\begin{array}{c}\text { Strain } \\
(\%)\end{array}$ & $\begin{array}{c}\varepsilon_{1} \\
(\mathrm{eV})\end{array}$ & $\begin{array}{c}\varepsilon_{2} \\
(\mathrm{eV})\end{array}$ & $\begin{array}{c}\varepsilon_{3} \\
(\mathrm{eV})\end{array}$ \\
\hline 0 & $0.167^{*}$ & 0.071 & 0.359 \\
1 & $0.161^{*}$ & 0.094 & 0.332 \\
2 & $0.156^{*}$ & 0.116 & 0.306 \\
3 & $0.150^{*}$ & 0.137 & 0.280 \\
4 & 0.143 & $0.157^{*}$ & 0.255 \\
5 & 0.134 & $0.175^{*}$ & 0.229 \\
\hline \hline
\end{tabular}

preferred diffusion path for $\mathrm{H}$ in bulk $\mathrm{Al}$. The various values of $\varepsilon_{3}$ as a function of tensile strain are also listed in Table II.

The calculated energy profiles allow us to estimate the bulk diffusion constant,

$$
D_{b}=\nu_{b} l_{b}^{2} \exp \left[-\varepsilon_{b} / k_{B} T\right]
$$

where $\nu_{b}$ and $l_{b}$ are the attempt frequency and hopping length for bulk diffusion. Approximating the energy differences near the equilibrium tetrahedral configuration by a second-order polynomial in the distance, we find an attempt frequency $\nu_{b}=0.8 \times 10^{11} \mathrm{sec}^{-1}$, while the hopping length between equivalent sites is $l_{b}=0.948 a$. The diffusion constant at room temperature $(300 \mathrm{~K})$ is estimated to be 1.78 $\times 10^{-11} \mathrm{~m}^{2} \mathrm{~s}^{-1}$ in the unstrained crystal. Assuming that the values of the attempt frequency and hopping length are not significantly affected by strain, the value of the bulk diffusion constant at room temperature and for $3 \%$ and $5 \%$ tensile strain is $3.46 \times 10^{-11} \mathrm{~m}^{2} \mathrm{~s}^{-1}$ and $1.31 \times 10^{-11} \mathrm{~m}^{2} \mathrm{~s}^{-1}$, respectively.

\section{Adsorption and diffusion of $\mathrm{H}$ on $\mathrm{Al}(111)$}

For $\mathrm{H}$ embrittlement of metals, it has been experimentally observed that the fracture surface is along the slip plane, where shear localization occurs. ${ }^{1,19}$ Apparently, adsorption and diffusion of $\mathrm{H}$ on the fresh fracture surface play an important role on the kinetics of crack propagation and the embrittling effect of $\mathrm{H}$. The critical energetics that are relevant to the adsorption and diffusion process not only can provide insight into the problem, they can also be used in an empirical analysis that can deal with the macroscopic aspects of this phenomenon. ${ }^{3}$

For this reason, we have considered the adsorption of a $\mathrm{H}$ atom on the (111) surface of $\mathrm{Al}$, in configurations corresponding to $1 \times 1, \sqrt{3} \times \sqrt{3}$, and $2 \times 2$ surface unit cells, or $\mathrm{H}$ coverages in the range $\Theta \in[0.25,1.0]$ ML. The $H$ atom was placed at the high-symmetry $F, H$, and $A$ points (see Fig. 1), as well as points at regular intervals between them, to determine the lowest energy configuration and the energy barrier for surface diffusion. By analogy to the definition of Eq. (1), the adsorption energy $E_{\mathrm{ad}}$ is defined as the energy of the configuration with a $\mathrm{H}$ atom on the (111) $\mathrm{Al}$ surface, relative 


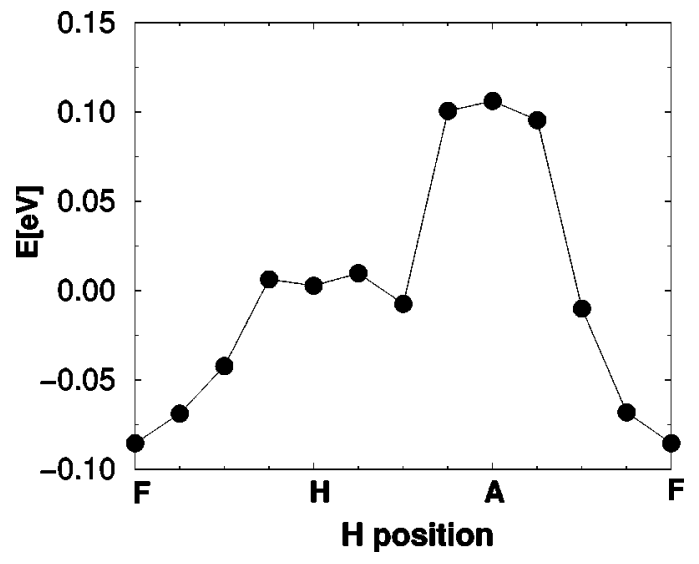

FIG. 5. Adsorption energy for $\mathrm{H}$ on the (111) Al surface within the $1 \times 1$ surface unit cell corresponding to $\mathrm{H}$ coverage $\Theta$ $=1 \mathrm{ML}$, for different positions in the [ $\overline{2} 11]$ direction: $F=\mathrm{fcc}$ site, $H=$ hcp site, $A=$ atop site.

to the same surface without $\mathrm{H}$ and using a gas of $\mathrm{H}_{2}$ molecules as a reservoir for the $\mathrm{H}$ atoms. For a full monolayer of $\mathrm{H}(\Theta=1.0)$ on the (111) Al surface, corresponding to the 1 $\times 1$ cell, the adsorption energy for the various positions along the $[\overline{2} 11]$ direction is given in Fig. 5 . The fcc site $(F)$ is the energetically preferred position with $E_{\text {ad }}$ $=-0.085 \mathrm{eV}$, whereas the hcp site $(H)$ has nearly zero adsorption energy, and the atop site $(A)$ is energetically unfavorable. We find that when displacing the $\mathrm{H}$ atom from the $F$ toward the $H$ and $A$ positions, the $\mathrm{Al}$ atoms near the surface are also displaced in the $[\overline{2} 11]$ direction, so as to maintain the high coordination of the $\mathrm{H}$ atom to the extent possible. This is especially pronounced at the bridge position, which is half way between the $F$ and the $H$ sites (see Fig. 1).

From the results of these calculations, we conclude that the diffusion of $\mathrm{H}$ on the (111) Al surface will follow a zig-zag path between successive $F$ and $H$ sites. We find that the energy difference between the $H$ and $F$ sites is a reasonable approximation for the diffusion energy barrier $\varepsilon_{s}$ within the numerical uncertainty inherent in the calculations. With this in mind, we have calculated the diffusion energy barrier as a function of $\mathrm{H}$ coverage, using multiples of the $1 \times 1$ surface unit cell that is given in Table III. It is clear from this table that the energy barrier for surface diffusion of an isolated $\mathrm{H}$ atom is approximately $0.163 \mathrm{eV}$, practically the same as that for bulk diffusion. We can also obtain an estimate of the surface diffusion constant using the energy as a function of $\mathrm{H}$ position from the calculation of the $1 \times 1$ unit cell:

$$
D_{s}=\nu_{s} l_{s}^{2} \exp \left[-\varepsilon_{s} / k_{B} T\right]
$$

where $\nu_{s}$ and $l_{s}$ are the attempt frequency and hopping length for surface diffusion. Using the same procedure as for bulk diffusion, we find an attempt frequency $\nu_{s}=0.7$ $\times 10^{10} \mathrm{sec}^{-1}$, the hopping length between equivalent sites is $l_{s}=0.707 a$, and the diffusion constant at room temperature $(300 \mathrm{~K})$ is $1.02 \times 10^{-12} \mathrm{~m}^{2} \mathrm{~s}^{-1}$.

Finally, we note that the presence of $\mathrm{H}$ on the (111) $\mathrm{Al}$ surface reduces the surface energy considerably. In order to quantify this observation, we report in Table III the calculated adsorption energy $E_{\text {ad }}$ for $\mathrm{H}$ atoms at various coverages. The reduction in surface energy in the presence of $\mathrm{H}$, $\Delta \gamma_{s}$, is obtained by converting the adsorption energy to a surface energy and subtracting from it the corresponding surface energy of pure Al. We find that this reduction in surface energy is a function of coverage, and it increases as the coverage decreases (Table III). Finally, we have performed one additional calculation in a larger $4 \times 4$ surface unit cell in order to determine to what extent this trend continues for lower coverages; the result is included in Table III. We conclude that the $\mathrm{H}$ coverage that gives the largest reduction in surface energy is in the range $0.0625 \leqslant \Theta \leqslant 0.25 \mathrm{ML}$.

It should be pointed out that $\Delta \gamma_{s}$ is a very important material parameter in determining the tendency of impurityinduced intergranular fracture. More specifically, according to the thermodynamic theory developed by Rice and Wang, 20 the potency of a segregating impurity in reducing the Griffith work of a brittle grain-boundary separation is a linear function of the difference $\Delta \gamma_{\mathrm{gb}}-\Delta \gamma_{s}$, that is, the difference between the segregation energy of the impurity at a grain boundary and at a free surface. A smaller reduction in surface energy (a less negative $\Delta \gamma_{s}$ ) indicates a weaker tendency for brittle intergranular fracture. Based on the fact that our calculated $\Delta \gamma_{s}$ is more than an order magnitude smaller than

TABLE III. Energy difference between the two high-symmetry positions $F$ and $H$ of a $\mathrm{H}$ atom on the $\mathrm{Al}(111)$ surface, identified as the surface diffusion activation energy $\varepsilon_{s}$; the adsorption energy $E_{\text {ad }}^{(F)}$ of $\mathrm{H}$ atoms at the energetically preferred fcc $(F)$ site on the (111) surface and the corresponding reduction in surface energy $\Delta \gamma_{s}$; the absorption energy $E_{\mathrm{ab}}^{(T)}$ of $\mathrm{H}$ atoms at the energetically preferred tetrahedral $(T)$ site in the interface between two (111) planes and the corresponding interface formation energy $\Delta \gamma_{\text {int }}$. All quantities are given as functions of $\mathrm{H}$ coverage $\Theta$ in monolayers (ML). The last line gives the corresponding results for the 32-atom bulk supercell of the conventional cubic cell.

\begin{tabular}{clccccc}
\hline \hline Supercell & $\begin{array}{c}\Theta \\
(\mathrm{ML})\end{array}$ & $\begin{array}{c}\varepsilon_{s} \\
(\mathrm{eV})\end{array}$ & $\begin{array}{c}E_{\mathrm{ad}}^{(F)} \\
(\mathrm{eV})\end{array}$ & $\begin{array}{c}\Delta \gamma_{s} \\
\left(\mathrm{~J} \mathrm{~m}^{-2}\right)\end{array}$ & $\begin{array}{c}E_{\mathrm{ab}}^{(T)} \\
(\mathrm{eV})\end{array}$ & $\begin{array}{c}\Delta \gamma_{\mathrm{int}} \\
\left(\mathrm{J} \mathrm{m}^{-2}\right)\end{array}$ \\
\hline $1 \times 1$ & 1.0 & 0.092 & -0.085 & -0.138 & +0.383 & +0.586 \\
$\sqrt{3} \times \sqrt{3}$ & 0.333 & 0.157 & -0.365 & -0.187 & +0.067 & +0.034 \\
$2 \times 2$ & 0.25 & 0.163 & -0.489 & -0.188 & -0.039 & -0.015 \\
$4 \times 4$ & 0.0625 & & -1.907 & -0.183 & & \\
$2 \times 2 \times 2$ & & 0.167 & & & -0.222 & \\
\hline \hline
\end{tabular}


typical values for intergranular fracture, ${ }^{20-22}$ we infer $\mathrm{H}$-induced fracture in $\mathrm{Al}$ to be of a transgranular nature. Of course, the definite determination of the tendency will also depend on $\Delta \gamma_{\mathrm{gb}}$, which is not available to us.

It is also instructive to compare this reduction in surface energy to the energy required to form $\mathrm{Al} / \mathrm{H} / \mathrm{Al}$ interface structures corresponding to the bulk unit cells with one $\mathrm{H}$ impurity discussed earlier. In Table III, we give the absorption energy $E_{\mathrm{ab}}$ obtained from the corresponding unit cells employed in the bulk calculations. As before, we use the pure Al bulk as a reference system and a gas of $\mathrm{H}_{2}$ molecules as the reservoir for $\mathrm{H}$ atoms. The configurations used for these calculations correspond to the formation of a planar interface between two (111) planes of bulk Al separated by an ordered layer of $\mathrm{H}$ atoms. We have calculated the interface formation energy $\Delta \gamma_{\mathrm{int}}$, as a function of $\mathrm{H}$ content at the interface, expressed in monolayer of $\mathrm{H}$; the results are given in Table III. Consistent with the calculations we presented earlier, this formation energy is positive for large $\mathrm{H}$ concentrations (recall the large positive absorption energy for a $\mathrm{H}$ atom in the $1 \times 1$ bulk supercell). However, with decreasing $\mathrm{H}$ concentration at the interface we expect that this formation energy will be reduced, and for small enough concentrations it should be negative, corresponding to the absorption energy for an isolated $\mathrm{H}$ atom in bulk $\mathrm{Al}$, which we found to be $-0.222 \mathrm{eV}$. Indeed, $\Delta \gamma_{\text {int }}$ becomes negative for the $2 \times 2$ supercell. Note that in this supercell the shortest distance between $\mathrm{H}$ atoms on the (111) plane is $a \sqrt{2}$, which is shorter than the distance between the $\mathrm{H}$ impurities in the 32-atom bulk supercell, equal to $2 a$.

\section{DISCUSSION AND CONCLUSIONS}

The following picture emerges from the calculations reported above. In a system consisting of a crystalline $\mathrm{Al}$ phase in equilibrium with a $\mathrm{H}_{2}$ gas, $\mathrm{H}$ atoms will be adsorbed on the (111) Al surfaces, the natural cleavage planes, in order to lower the surface energy. The equilibrium coverage will be in the range $0.0625 \leqslant \Theta \leqslant 0.25 \mathrm{ML}$. Diffusion of $\mathrm{H}$ on the surface and in the bulk is relatively fast. Using the values for the diffusion constants we determined earlier, we find that at room temperature the length scale for surface diffusion in a time interval of $1 \mathrm{sec}$ is $\sim 0.3 \mu \mathrm{m}$, while for bulk diffusion it is $\sim 7 \mu \mathrm{m}$. Over such distances the equilibrium surface $\mathrm{H}$ coverage and bulk $\mathrm{H}$ content will be dictated by thermodynamic considerations. The calculated bulk absorption energy for the tetrahedral site, $E_{\mathrm{ab}}^{(T)}=-0.222 \mathrm{eV}$, indicates that energetically it is possible for $\mathrm{H}$ to end up in the bulk. However, this is not the preferred configuration. In fact, the calculated surface adsorption energies per $\mathrm{H}$ atom are lower than the bulk absorption energies. For example, from the calculation of the $4 \times 4$ surface unit cell with one $\mathrm{H}$ atom at the $F$ position, we find a surface adsorption energy $E_{\text {ad }}^{(F)}$ $=-1.907 \mathrm{eV}$, significantly lower than the bulk absorption energy. In this configuration the $\mathrm{H}$ atom can be considered as an isolated atom on the $\mathrm{Al}$ surface. It appears from these calculations that if there is any $\mathrm{H}$ in bulk $\mathrm{Al}$ and if the system is allowed to equilibrate with the surface, $\mathrm{H}$ tends to diffuse out and remains on the surface. This conclusion rests on the assumption that a clean and atomically flat (111) Al surface is available, which is usually not the case in reality due to the tendency of $\mathrm{Al}$ surfaces to oxidize. The presence of an oxide on the surface will completely alter the thermodynamic balance, making it possible for $\mathrm{H}$ atoms to remain in the bulk. Moreover, we have not investigated the possibility of surface vacancies or other defects on the surface, which can also change the thermodynamic picture. The presence of such defects can only lower the $\mathrm{Al}$ surface energy, while the binding of $\mathrm{H}$ atoms at such defects may not be preferred over binding on the flat surface, as the values of $E_{\mathrm{ab}}^{(T)}$ and $E_{\mathrm{ad}}^{(F)}$ reported in Table III suggest. Thus, the presence of defects on the $\mathrm{Al}$ surface may also suffice to make the incorporation of $\mathrm{H}$ atoms in the bulk thermodynamically stable.

On the other hand, our calculations for $\mathrm{H}$ in bulk $\mathrm{Al}$ show that $\mathrm{H}$ atoms feel an effective repulsive interaction for distances shorter than twice the primitive lattice constant, $a / \sqrt{2}$. This is evident from the $\mathrm{Al} / \mathrm{H} / \mathrm{Al}$ interface energies $\Delta \gamma_{\text {int }}$, reported in Table III, which are all positive except for the $2 \times 2$ supercell. Therefore, $\mathrm{H}$ atoms in bulk $\mathrm{Al}$ cannot form dense clusters but have to be apart from each other by a distance at least $a / \sqrt{2}$. This result is significant because it spells out the importance of $\mathrm{H}-\mathrm{H}$ interactions and casts doubt on studies that ignore such interactions. The energetically favorable configuration that we found for high $\mathrm{H}$ concentrations in the bulk, with a $\mathrm{H}$ atom between two $\mathrm{Al}$ atoms directly above and below it in the [111] direction, also has interesting implications. Since this structure has a lower energy than the undistorted crystal configuration with equal $\mathrm{H}$ concentration but also involves a large volume relaxation $(18 \%)$, we conclude that if there are voids or other defects that give rise to tensile strain, $\mathrm{H}$ atoms will be preferentially bound to those sites, such as the cores of edge dislocations. In fact, a recent study showed that the binding energy of $\mathrm{H}$ to the core of an edge dislocation is much larger than that of a screw dislocation, ${ }^{2}$ which is in line with our observation here. These results have an important consequence for dislocation motion: the edge dislocation needs to turn into a screw dislocation in order to cross slip, a process that will be hindered by the binding of $\mathrm{H}$ atoms to the edge dislocation, consistent with experimental observations. ${ }^{23}$ More importantly, this H-inhibited cross slip will give rise to slip planarity and possibly, shear localization, the two most important elements to understand $\mathrm{H}$ embrittlement in terms of the HELP mechanism. ${ }^{1}$

Finally, we turn our attention to the effects of $\mathrm{H}$ on the intrinsic stacking fault energy $\gamma_{\mathrm{sf}}$, unstable stacking fault energy $\gamma_{\mathrm{us}}$, and cleavage energy $\gamma_{\mathrm{cl}}$. As already noted, the presence of $\mathrm{H}$ reduces all these quantities relative to their values in pure $\mathrm{Al}$. The ratio $\gamma_{\mathrm{cl}} / \gamma_{\mathrm{us}}$ as a function of impurity content has been employed to discern brittle vs ductile response. ${ }^{24}$ The simple physical picture behind this argument is that a low value of this ratio indicates a preference for cleavage rather than dislocation generation at a crack tip that is controlled by the value of $\gamma_{\mathrm{us}}{ }^{14}$ this behavior is associated with brittle failure. Conversely, a high value of this ratio indicates the preference for dislocation generation at a crack tip, a behavior associated with ductile response. While this picture may be overly simplistic for quantitative analysis, it 
does give insight about general trends and can even lead to useful predictions that have been verified experimentally. ${ }^{24}$ For $\mathrm{H}$ in bulk $\mathrm{Al}$, this ratio is higher than the value in pure $\mathrm{Al}$ except at the highest $\mathrm{H}$ concentration considered here (14.3 at. \%, corresponding to the $1 \times 1$ supercell), clearly suggesting that $\mathrm{H}$ embrittlement in $\mathrm{Al}$ takes place as plastic rupture rather than as brittle separation. ${ }^{1}$ In fact, recent work ${ }^{2}$ indicates that $\mathrm{H}$ in $\mathrm{Al}$ can indeed lead to enhanced local plasticity, a precursor to $\mathrm{H}$ embrittlement. ${ }^{1}$

The lower value of the intrinsic stacking fault energy in the presence of $\mathrm{H}$ suggests a larger separation of the partial dislocations in $\mathrm{Al}$, which could hinder the dislocation cross slip since the partial dislocations must be constricted before cross slip can take place. But a more detailed analysis based on the Peierls-Nabarro model ${ }^{2}$ shows that, even though the stacking fault energy is lowered by the presence of $\mathrm{H}$, the partial dislocations are not split any further than in pure $\mathrm{Al}$, while the core width of the dislocations is increased significantly giving rise to enhanced dislocation mobility.

To summarize, we have performed density-functional theory calculations to study the energetics of $\mathrm{H}$ impurities in bulk and on the (111) surface of Al. We have obtained the dependence of the stacking fault energy and the cleavage energy, as well as the $\mathrm{Al} / \mathrm{H}$ surface energy and the $\mathrm{Al} / \mathrm{H} / \mathrm{Al}$ interface formation energy, on $\mathrm{H}$ concentration. The results indicate that there is a strong dependence of the GSF energy in the $[\overline{2} 11]$ direction, the cleavage energy in the [111] direction and the $\mathrm{Al} / \mathrm{H} / \mathrm{Al}$ interface formation energy, on $\mathrm{H}$ concentration and on tensile strain. We are able to explain the $\mathrm{H}$-induced reduction of the stacking fault energy and cleavage energy in $\mathrm{Al}$ from an electronic structure point of view, and conjecture that such reduction can also take place in other H-metal systems. It is found that the dependence of the $\mathrm{Al} / \mathrm{H}$ surface energy on the $\mathrm{H}$ coverage is less pronounced, with the optimal coverage being $\leqslant 0.25 \mathrm{ML}$. The calculated activation energy for diffusion between high-symmetry sites in the bulk and on the surface is practically the same, 0.167 $\mathrm{eV}$, in good agreement with experimental measurements. Although our calculations reported here provide strong theoretical evidence for the HELP mechanism, they are not able to answer how HELP eventually leads to $\mathrm{H}$ embrittlement. Nevertheless, we believe that our work sets the stage for developing a comprehensive theory of $\mathrm{H}$ embrittlement, which most likely will necessitate a multiscale framework.

\section{ACKNOWLEDGMENTS}

We would like to thank Nick Choly, Emily Carter, Rob Phillips, and Bill Curtin for useful discussions. We acknowledge the use of computer facilities at Brown University. This work was funded by AFOSR, Contract No. F49620-99-10272 .
${ }^{1}$ S. M. Myers, M. I. Baskes, H. K. Birnbaum, J. W. Corbett, G. G. DeLeo, S. K. Estreicher, E. E. Haller, P. Jena, N. M. Johnson, R. Kirchheim, S. J. Pearton, and M. J. Stavola, Rev. Mod. Phys. 64, 559 (1992), and references therein.

${ }^{2}$ G. Lu, Q. Zhang, N. Kioussis, and E. Kaxiras, Phys. Rev. Lett. 87, 095501 (2001).

${ }^{3}$ O. Nguyen and M. Ortiz (unpublished).

${ }^{4}$ M. Robertson, Eng. Fract. Mech. 64, 649 (1999).

${ }^{5}$ H. K. Birnbaum and P. Sofronis, Mater. Sci. Eng., A 176, 191 (1994).

${ }^{6}$ G. Kresse and J. Furthmüller, Phys. Rev. B 54, 11169 (1996).

${ }^{7}$ D. Vanderbilt, Phys. Rev. B 41, 7892 (1990).

${ }^{8}$ D. M. Ceperley and B. J. Alder, Phys. Rev. Lett. 45, 566 (1980).

${ }^{9}$ J. P. Perdew and A. Zunger, Phys. Rev. B 23, 5048 (1981).

${ }^{10}$ H. J. Monkhorst and J. D. Pack, Phys. Rev. B 13, 5188 (1976).

${ }^{11}$ F. Birch, J. Geophys. Res. B 83, 1257 (1978).

${ }^{12}$ J. P. Hirth and J. Lothe, Theory of Dislocations (Wiley, New York, 1992).

${ }^{13}$ M. S. Duesbery, Modell. Simul. Mater. Sci. Eng. 6, 35 (1998).

${ }^{14}$ J. R. Rice, J. Mech. Phys. Solids 40, 239 (1992); J. R. Rice, G. E. Beltz, and Y. Sun, Topics in Fracture and Fatique, edited by A. Argon (Springer, Berlin, 1992).
${ }^{15}$ G. Lu, N. Kioussis, V. V. Bulatov, and E. Kaxiras, Phys. Rev. B 62, 3099 (2000).

${ }^{16}$ Y. Sun and E. Kaxiras, Philos. Mag. A 75, 1117 (1997).

${ }^{17}$ M. Hashimoto and R. M. Latanision, in Chemistry and Physics of Fracture, edited by R. M. Latanision and R. Jones (Martinus Nijhoff, Dordrecht, The Netherlands, 1987), p. 505.

${ }^{18}$ G. A. Young and J. R. Scully, Acta Mater. 46, 6337 (1998).

${ }^{19}$ T. Matsumoto and H. K. Birnbaum, Trans. Jpn. Inst. Met. 21, 493 (1980); S. P. Lynch, J. Mater. Sci. 21, 692 (1986).

${ }^{20}$ J. R. Rice and J. S. Wang, Mater. Sci. Eng., A 107, 23 (1989).

${ }^{21}$ G. Lu, N. Kioussis, R. Wu, and M. Ciftan, Phys. Rev. B 59, 891 (1999).

${ }^{22}$ R. Wu, A. J. Freeman, and G. B. Olson, Science 265, 376 (1994).

${ }^{23}$ P. J. Ferreira, I. M. Robertson, and H. K. Birnbaum, Acta Mater. 47, 2991 (1999).

${ }^{24}$ U. V. Waghmare, E. Kaxiras, V. Bulatov, and M. S. Duesbery, Modell. Simul. Mater. Sci. Eng. 6, 483 (1998); U. V. Waghmare, V. Bulatov, E. Kaxiras, and M. S. Duesbery, Mater. Sci. Eng., A 261, 147 (1999); U. V. Waghmare, E. Kaxiras, V. Bulatov, and M. S. Duesbery, Philos. Mag. A 79, 655 (1999); U. V. Waghmare, E. Kaxiras, and M. S. Duesbery, Phys. Status Solidi B 217, 545 (2000). 